Editorial

\title{
Etiological study of neonatal septicaemia
}

\author{
Dhanjal G.S. ${ }^{1}$, Agrawal S. ${ }^{2}$, Sharda P. ${ }^{3}$ \\ ${ }^{1}$ Dr. Gurdeep S Dhanjal, Professor of Pediatrics, Maharishi Markandeshwar Institute of Medical Science and Research, \\ Mullana, Ambala, Harayana. ${ }^{2}$ Dr. Sonam Agrawal, Senior Resident, Maharishi Markandeshwar Institute of Medical \\ Sciences and Research, Mullana, Ambala, Harayana. ${ }^{3}$ Dr. Prateek Sharda, Senior Resident, Maharishi Markandeshwar \\ Institute of Medical Sciences and Research, Mullana, Ambala, Harayana. India.
}

Corresponding Author: Dr. Sonam Agrawal, Senior Resident, MMIMSR, Mullana Ambala. E-mail: dr.sonamagrawal@gmail.com

\begin{abstract}
Introduction: Neonatal sepsis is a clinical syndrome of bacteremia characterized by systemic signs and symptoms of infection in the first month of life.Our aim was to study the etiological profile of neonatal septicaemia and their sensitivity pattern. Methodology: The study was conducted over a period of one and half years in the Neonatal Intensive Care Unit (NICU), MMIMSR, Mullana, Ambala, Haryana. A total of 100 cases with positive sepsis screen were identified with standard biochemical tests and these newborns were subjected to blood culture and antimicrobial susceptibility test. Results: There were 66 male babies and 34 female babies with male: female ratio of 1.9:1. In the study $94 \%$ cases were early onset neonatal sepsis and $6 \%$ were late onset neonatal sepsis. The blood cultures of 54 babies showed growth, out of these $16(29.6 \%)$ cases were grampositive bacteria, 34(62.9\%) were gram negative bacteria and 4(7.4\%) showed fungal growth (i.e. candida albicans). Acinetobacter species were most common among gramnegative organisms i.e. $64.7 \%$. Staphylococcus aureus along with coagulase-negative staphylococci (CONS) were common organisms seen among grampositive bacteria. Among gramnegative isolates $62.9 \%$ were extended spectrum beta lactamase (ESBL) producers. Conclusion: Neonatal septicaemia is a life-threatening emergency. The study of etiological profile and their antibiotic sensitivity pattern plays a significant role in decreasing the neonatal mortality rate. The rational use of antibiotics will reduce infection rate ensuring better therapeutic success and reduce the resistance of the organism to available antibiotics.
\end{abstract}

Keywords: Neonatal septicaemia, Blood culture, Sepsis, Antimicrobial susceptibility

\section{Introduction}

Neonatal sepsis is a clinical syndrome of bacteremia characterized by systemic signs and symptoms of infection in the first month of life [1]. It comprises meningitis, pneumonia, arthritis, osteomyelitis, and urinary tract infections [2]. This excludes local infection of newborn such as omphalitis, pyoderma and conjunctivitis [3]. It is estimated that $20 \%$ of neonates develop sepsis and approximately $1 \%$ deaths are related to sepsis [4].

Neonatal septicemia is by far the most fatal sequelae of such infections [5]. For epidemiological and therapeutic purpose, neonatal septicaemia is categorized into early onset septicaemia (presents within the first 72 hours of life) and late onset septicaemia (presents after 72 hours of life) [2].

Manuscript received: $30^{\text {th }}$ December 2017

Reviewed: $10^{\text {th }}$ January 2018

Author Corrected: $18^{\text {th }}$ January 2018

Accepted for Publication: $23^{\text {rd }}$ January 2018
The gold standard for the diagnosis of neonatal sepsis is a positive blood culture $[2,6]$. Definitive culture results take at least $48-72 \mathrm{hrs}$ resulting in treatment delays.

But with improved bacteriological techniques such as BACTEC and BACT/ALERT blood culture system can detect bacteria at a concentration of 1-2 colony forming unit (cfu)/ $\mathrm{ml}[2,7]$. Early diagnosis and treatment with appropriate antibiotics would minimize the risk of severe morbidity and mortality besides reducing the emergence of multiple resistant organisms in nurseries by rational antibiotics use. Periodic evaluation of organisms causing neonatal sepsis is essential for the appropriate management of neonates.

Therefore, the present study was undertaken to determine the etiological profile and the antibiotic sensitivity pattern of the microbial isolates in a tertiary level hospital. 
Editorial

\section{Material and Methods}

The study was a prospective observational study conducted in neonatal intensive care unit (NICU) of Maharishi Markandeshwar institute of medical sciences and research (MMIMSR), Mullana, Ambala over a period of one and half year (November 2013 to May 2015). Institutional ethical clearance was taken for the study.

Neonates admitted in the hospital with maternal risk factors were subjected to a battery of test collectively called the "sepsis screen". Parameters included in the sepsis screen and their abnormal values were total leukocyte count (TLC) $<5000 /$ cumm, absolute neutrophil count $(\mathrm{ANC})<1800 / \mathrm{cumm}$, immature to mature neutrophil ratio $>0.2$, micro ESR $>15 \mathrm{~mm}$ in $1^{\text {st }}$ hour and C-reactive protein $(\mathrm{CRP})>1 \mathrm{mg} / \mathrm{dl}$. Sepsis screenwas considered positive when two or more parameters were abnormal.

Neonates with positive sepsis screen were included in the study. Babies whose parent did not gave consent were excluded from the study. 100 newborns satisfying the inclusion criteria were enrolled in the study.

With all aseptic precautions $2 \mathrm{ml}$ blood was taken in EDTA vacutainer and was processed for TLC, ANC, peripheral blood smear (PBS) for I:T ratio and micro ESR. Also $1 \mathrm{ml}$ blood sample was taken for estimation of a qualitative CRP result.

Auto hematology analyzer ( 3 parts) was used to analyze the sample. TLCwas calculated by direct counting of leukocytes in an improved Neubauer's chamber. ANC was calculated on PBS stained by Leishman's stain.
Neutrophils were classified as band forms when there are no nuclear segmentation or when the width of the nucleus at any constriction was not less than one-third the width at its widest portion. Band forms together with less mature cell forms were classified as immature polymorphonuclear (PMN) leukocytes [8]. Immature to total neutrophil ratio was calculated on PBS stained by Leishman's stain. Micro ESR was performed using standard preheparinised micro hematocrit tubes $(75 \mathrm{~mm}$ in length with internal diameter $1.1 \mathrm{~mm}$ and outer diameter of $1.5 \mathrm{~mm}$ ) and by recording the fall of erythrocyte column after 1 hour. [8].

Another $2 \mathrm{ml}$ blood collected with all aseptic precautions was inoculated into BACT/ALERT RPF (Biomerieux, INC. Durhams, NC 27704) containing $20 \mathrm{ml}$ of broth. The blood and broth were mixed gently and bottles were transported to laboratory for incubation in BACT/ALERT 3D system and further processing was done as per manufacturer's guidelines.

Interpretation: Those blood culture indicated positive or negative by BACT/ALERT 3D system were sub cultured on sheep blood agar and Mac Conkey agar. The sheep blood agar and Mac Conkey's medium were incubated at $35+2^{\mathrm{O}}$ Celsius for 18-24 hours in aerobic conditions. Various organisms were identified on the basis of colony morphology and standard biochemical tests. Those blood culture bottles which were negative for 5 days (as setting of BACT/ ALERT 3D system) were reported as "no growth". The isolates were subjected to antimicrobial susceptibility testing by Kirby Bauer disk diffusion method as per clinical and laboratory standard institute (CLSI) Guidelines2011 (9).

\section{Results}

In the present study, total of 100 cases with significant sepsis score were studied and blood culture were performed. Among these 90 cases (i.e. 90\%) were of early onset neonatal septicaemia and 10 (i.e. 10\%) cases were of late onset sepsis. In the present study the ratio of male: female is $1.9: 1$. Out of 100 babies $62 \%$ babies were preterm and $38 \%$ were term with preterm to term ratio of 1:1.6.

Out of 100 neonates, blood culture was positive in $54(54 \%)$ cases where as in $46(46 \%)$ cases blood culture was negative. Among the 54 blood culture positive cases gram negative bacteria were present in $34(63 \%)$ cases followed by gram positive bacteria in $16(29.6 \%)$ cases and fungal growth in $4(7.4 \%)$ cases (TABLE 1$)$

Acinetobacter was the commonest pathogen found in blood culture followed by citrobacter. Only $37.5 \%$ isolates of Staphylococcus aureus and Staphylococcus epidermidis respectively were sensitive to penicillin and oxacillin. Methicillin resistance was seen in $25 \%$ cases of Staphylococcus aureus.

All gram-positive cocci were sensitive to vancomycin. The enterobacteriacae family showed high resistance to various antimicrobial agents like ampicillin, gentamycin, cefazolin, cefoperazone, cefotaxime and ceftriaxone. Most of them had good sensitivity to amikacin, ciprofloxacin, ceftazidime and piperacillin tazobactum. 
Pseudomonas aeruginosa and acinetobacter species showed high resistance to various antimicrobial agents like cefepime, cefoperazone, cefotaxime and ceftazidime. Most of the gram negative organism had moderate sensitivity to amikacin, piperacillin, tazobactum and good sensitivity to meropenem and imipenem.

We observed that meropenem and imipenem were highly effective antibiotic for infections with multidrug resistant gramnegative bacilli. All the four cases with positive candida growth were sensitive to fluconazole.

Table-1: Microbial profile of neonatal septicaemia.

\begin{tabular}{|c|c|c|}
\hline Organisms & $\begin{array}{c}\text { Cases (54/100) } \\
\text { (blood culture positive) }\end{array}$ & Drug sensitivity \\
\hline \multicolumn{3}{|c|}{ Gram-positive : 16 cases } \\
\hline Staph aureus & $6(37.5 \%)$ & Penicillin, oxacillin, vancomycin, linezolid \\
\hline MRSA & $4(25 \%)$ & Vancomycin, linezolid \\
\hline CONS (S. epidermidis) & $6(37.5 \%)$ & Penicillin, oxacillin, vancomycin, linezolid \\
\hline Acinetobacter & Gram-negative: 34 cases & $\begin{array}{c}\text { amikacin,ciprofloxacin,piperacillin, } \\
\text { tazobactum, meropenem,imipenem }\end{array}$ \\
\hline Citrobacter & $22(64.7 \%)$ & $\begin{array}{c}\text { amikacin,ciprofloxacin, ceftazidime, } \\
\text { piperacillin, tazobactum, meropenem \& } \\
\text { imipenem }\end{array}$ \\
\hline Pseudomonas & $8(23.5 \%)$ & Piperacillin,tazobactum, Meropenem and \\
& & \\
\hline Candida albicans & $4(11.7 \%)$ & Fungi: 4 cases \\
\hline
\end{tabular}

\section{Discussion}

The advancement in neonatal intensive care medicine is a double edge sword, with neonatal survival improvement on one side and increased rate of long term morbidity on another. The microorganism pattern of neonatal sepsis is different at different hospitals and its pattern changes with time hence periodic reevaluation of the etiological agent is useful in the management of neonatal sepsis. Despite all efforts, a rapid sensitive diagnostic tool for neonatal septicaemia is yet to be found.

Number of studies have been done on the risk factors, etiology, haematological parameters and on the clinical profile of neonatal septicaemia. Blood culture is the gold standard for definitive diagnosis of neonatal septicemia, but it has its own limitations as it requires a well equipped laboratory, has a success rate of $40 \%$, very time consuming, and may give spurious positive results. The results of blood culture may take about a week, necessitating initial empirical treatment of suspected septicemia. Overall incidence of culture proven sepsis varies between 1-8 cases per 1000 live births with almost equal distribution of early onset and late onset cases.
This present study was undertaken to study the etiological profile in newborn with positive sepsis screen so that prompt therapy be instituted to reduce morbidity and mortality. The most common agents causing neonatal sepsis are bacteria and only a proportion of the blood culture from cases with clinical sepsis will show growth of organism.

In our study the male to female ratio was $1.9: 1$ which is similar to the findings by Jain NK et al (2:1) [10] and by Jia-hong Jiang et al (1.4:1) [11]. Probably the ratio in favour of male could be because of the priority given to the male babies for medical care in our society.

In our study $90 \%$ of the cases were of early onset neonatal sepsis whereas late onset sepsis was seen in $10 \%$ cases. The large number of cases of EONS may be because our tertiary care hospital has a well-established maternity wards and caters rural population which do not have good health servicesespecially NICU leading to early referral to our institute.

In the present study the blood culture positivity rate was $54 \%$, which is similar with other studies $[12,13]$. The 
Editorial

frequency of isolation of gram positive and gram negative bacteria from blood culture in our study was $29.6 \%$ and $63 \%$ of culture positive cases respectively. This finding is almost similar to that of other studies done by Ahmed et al [14] and Kapoor et al [15] who foundgram-negative bacilli in $70 \%$ and $62 \%$ of culture positive neonates respectively. In the study done by Simiyu et al [16]66.6\% of culture positive cases were gramnegative organisms and 33.4\% were gram-positive. In the study by Agnihotri et al[17] $58.5 \%$ of positive culture showed growth of gramnegative organisms.

Acinetobacter and Citrobacter (gram negative organisms) were the two most common bacteria isolated from culture in our study. Most of the studies done earlier also shows predominance of gram negative organisms. The predominance of Acinetobacter as the causative agent of neonatal sepsis may be due to the selective pressure of antimicrobial agents so that resistant organisms tend to colonize and proliferate in the neonates.

In the present study majority of gram-negative bacilli i.e. Acinetobacter and Citrobacter isolates were resistant to all the commonly used antibiotics except meropenem, imipenem, piperacillin, tazobactum and amikacin. In another study from North India, 30-80\% of the gram negative isolates were resistantto thirdgeneration cephalosporins [18]. As amikacin shows good activity against gram-negative bacteria it should preferably beincluded in empirical regimen while third generation cephalosporins should not be used alone.

\section{Conclusion}

Neonatal septicaemia is a life-threatening emergency. The study of etiological profile and their antibiotic sensitivity pattern plays a significant role in decreasing the neonatal mortality rate. Blood culture results takes at least 48-72 hrs which result in treatment delays causing an increase in neonatal mortality.

Hence knowing the changing pattern of microorganism with studies done at intervals would help to decrease the neonatal mortality with appropriate use of antibiotics along with good infection control practices.

Funding: Nil, Conflict of interest: None initiated, Perission from IRB: Yes

\section{References}

1. Aggarwal R, Sarkar N, Deorari AK, Paul VK. Sepsis in the newborn. Indian J Pediatr. 2001 Dec;68(12): 1143-7.
2. Sankar MJ, Agarwal R, Deorari AK, Paul VK. Sepsis in the newborn. Indian J Pediatr. 2008 Mar; 75 (3): 261-6.

3. Paul VK and Singh MB. Neonatal sepsis in : Medical Emergencies in Children. Meharban Singh, $3^{\text {rd }}$ edition, Sagar Publications, New Delhi 2000: 117-35.

4. Begum S et al. Bacteriological Profile of Neonatal Sepsis in a Tertiary Hospital in MervatG. Al-Inany. Journal of Bangladesh college of Physicians and Surgeons. 2012;30(2):66-70.

5. Naik SA, Met gnd SC, Desai A, Patil CS. Bacteriological Profile of patient with neonatal septicaemia- A hospital based cross sectional study. Indian Journal of Maternal and Child Health 2012, AprJun; 14 (2): 1-6.

6. Multu M, Aslan Y, Saygin B, Yilmaz G, Bayramo LU G, Köksal I. Neonatal Sepsis Caused by Gramnegative Bacteria in a Neonatal Intensive Care Unit: A Six Years Analysis .HK J Paediatr (New series) 2011; 16 (4): 253-257.

7. Bhat RY, Leslie ES Lewis and Vandana KE. Bacterial isolates of early onset neonatal sepsis and their antibiotic susceptibility pattern between 1998 and 2004: An audit from centre in India. Italian Journal of Pediatrics 2011;37:32.

8. Singh M. Care of the newborn. $7^{\text {th }}$ ed. New Delhi: Sagar publications;2010;223-233.

9. CLSI. Performance standards for Antimicrobial Susceptibility testing; twenty first Informational Supplement. CLSI document M100-S21. Wayne, PA: Clinical and Laboratory standards Institute;2011.

10. Jain NK, Jain VM, Maheshwari S. Clinical profile of neonatal sepsis. Kathmandu Univ Med J (KUMJ). 2003 Apr-Jun;1(2):117-20.

11. Jiang JH, Chiu NC, Huang FY, Kao HA, Hsu CH, Hung HY, Chang JH, Peng CC. Neonatal sepsis in the neonatal intensive care unit: characteristics of early versuslateonset. J Microbiol Immunol Infect. 2004 Oct; 37 (5):301-6.

12. Roy I, Jain A, Kumar M, Agarwal SK. Bacteriology of neonatal septicaemia in a tertiary care hospital of northern India. Indian J Med Microbiol. 2002 Jul-Sep; 20 (3):156-9. 


\section{Editorial}

13. Sriram R. Correlation of blood culture results with the sepsis screen in the diagnosis of neonatal septicaemia. Int J Bio Med Res 2011;2(1):360-368.

14. Ahmed AS, chowdhury MA, Hoque M, Darmstadt GL. Clinical and bacteriological profile of neonatal septicaemia in a tertiary level pediatric hospital in Bangladesh. Indian Pediatr.2002;39:1034-9.

15. Kapoor L, Randhawa VS, Deb M. Microbiological profile of neonatal septicemia in a pediatric care hospital in Delhi. J Commun Dis. 2005 Sep; 37 (3): 227-32.
16. Simiyu DE. Neonatal septicaemia in low birth weight infants at Kenyatta National Hospital, Nairobi. East Afr Med J. 2005 Mar; 82(3):148-52.

17. Agnihotri N, Kaistha N, Gupta V. Antimicrobial susceptibility of isolates from neonatal septicemia. Jpn J Infect Dis. 2004 Dec;57(6):273-5.

18. Kaistha N, Mehta M, Singla N, Garg R, Chander J. Neonatal Septicaemia isolates and resistance patterns in atertiary hospital of North India. J Infect Dev Ctries. 2009; 4:55-7.

\section{How to cite this article?}

Dhanjal G.S, Agrawal S, Sharda P. Etiological study of neonatal septicaemia. Int J Pediatr Res. 2018;5(1):19-23. doi:10.17511/ijpr.2018.i01.05. 